\title{
Alcoholic Hepatitis and Intestinal Barrier Breakdown: A Theoretical Reappraisal Based on Pentoxifylline's Action
}

\author{
Stelios F. Assimakopoulos ${ }^{\mathrm{a}, \mathrm{c}}$, Konstantinos C. Thomopoulos ${ }^{\mathrm{b}}$, \\ Chrisoula Labropoulou-Karatza ${ }^{a}$
}

Alcoholic hepatitis is one of the most serious forms of alcoholic liver injury, associated with significant early mortality, as inpatient mortality can attain $50-75 \%$ in the most severe forms [1,2]. The treatment of alcoholic hepatitis remains one of the main challenges to clinicians involved in the management of severe alcoholic liver disease and represents one of the most debated topics in medicine and a field of continued research.

While the histology of alcoholic hepatitis is well characterized, its pathogenesis has not been clearly explained and is an era of active research. A number of hepatocellular and inflammatory processes including the potential involvement of innate and adaptive immunologic responses, oxidative stress and impaired hepatic regeneration are under investigation $[3,4]$. On this basis, diverse treatment options (glucocorticoids, pentoxifylline, infliximab, s-adenosyl-methionine and colchicine) have been tried in alcoholic hepatitis with a common goal of blocking the release of chemokines and cytokines by inflammatory cells in response to alcoholassociated oxidant stress and endotoxemia [5].

Current guidelines recommend the use of glucocorticosteroids for patients with severe alcoholic hepatitis as defined by the Maddrey score (discriminant-function $\geq 32$ ) $[5,6]$. However, their use is limited in cases of gastrointestinal bleeding or active infection. Amongst other available treatment options that have already been tested, only pentoxifylline (PTX) may be considered as an alternative to corticosteroids for severe acute alcoholic hepatitis based on the favourable results of studies comparing PTX with placebo $[7,8]$. Although primary treatment of severe alcoholic hepatitis with PTX is not currently recommended due to lack of evidence of its efficacy as compared to the standard treatment with corticosteroids, a very recent study by De BK et al [9] compared the two treatments head to head in a random-

Manuscript accepted for publication April 27, 2009.

aDepartment of Internal Medicine, University Hospital of Patras, Patras 26504, Greece

${ }^{b}$ Division of Gastroenterology, Department of Internal Medicine, University Hospital of Patras, Patras 26504, Greece

cCorresponding author: sassim@upatras.gr

doi: $10.4021 /$ gr2009.05.1288 ized controlled manner and demonstrated the superiority of PTX over corticosteroids, in terms of patients' survival and risk-benefit profile.

Up to now, the use of PTX in severe alcoholic hepatitis has been supported by three clinical studies. The first one was conducted by McHutchison et al in 1991, in patients with severe alcoholic hepatitis (defined as DF score $\geq 32$ ) and showed that PTX reduced the development of hepatorenal syndrome, and as a consequence mortality, in comparison to patients who received placebo [7]. These findings were confirmed in 2000 by Akriviadis et al [8] in a double-blind placebo-controlled trial. In the latter study, twenty-four percent of PTX treated patients and $46.1 \%$ of control patients died during hospitalization. The survival benefit of PTX appeared to be related to a significant reduction of hepatorenal syndrome. Among patients who died, hepatorenal syndrome had developed in $50 \%$ of PTX patients and in $91.7 \%$ of placebo patients. The third and very recent study was conducted by De BK and colleagues [9], in a randomized double-blind controlled study, the efficacy of PTX was evaluated over the standard treatment of prednisolone in the management of severe alcoholic hepatitis (DF $\geq 32$ ). This study showed a significantly reduced mortality among patients in the PTX group (14.71\%) compared to those receiving prednisolone (35.29\%, P $=0.04)$, accompanied by an advantageous safety profile. Reduction of mortality in the PTX group was again related to a significant reduction of hepatorenal syndrome. Among patients who died, hepatorenal syndrome had developed in $50 \%$ of prednisolone treated patients and in none of PTX treated patients.

The above stated studies have demonstrated that survival advantage in PTX treated patients with severe alcoholic hepatitis is clearly related to prevention of hepatorenal syndrome [7-9]. The mechanism of this beneficial effect is not evident by available data and only speculations could be made on this matter. PTX, a non-specific phosphodiesterase inhibitor, is a suppressor of tumor necrosis factor alpha (TNF- $\alpha$ ), prevents leukocyte adherence to vascular endothelium, down-regulates the expression of intercellular adhesion molecule-1 in monocytes and improves the rheological properties of blood [10]. The rationale of its use in alcoholic hepatitis is based on its property to inhibit the synthesis of 
TNF- $\alpha$, which is considered as a pivotal mediator of liver injury in alcoholic hepatitis [4, 8]. However, prevention of hepatorenal syndrome and survival advantage in patients with severe alcoholic hepatitis treated with PTX was not associated with decreased circulating TNF- $\alpha$ levels or with improved liver function [8]. These findings point towards an alternative explanation of PTX's positive effect on renal function in alcoholic hepatitis, but before any assumption is made on this issue it is essential to review the pathophysiology of hepatorenal syndrome with a special focus on alcoholic hepatitis-associated hepatorenal syndrome.

Hepatorenal syndrome is defined as a specific type of functional renal failure complicating advanced liver disease (e.g. decompensated cirrhosis, alcoholic hepatitis, or acute liver failure). The pathogenesis of this syndrome is the result of an extreme underfilling of the arterial circulation secondary to an arterial vasodilation located in the splachnic circulation. This underfilling triggers a compensatory response with activation of the sympathetic nervous system and release of humoral and renal vasoactive mediators leading to intense renal vasoconstriction, especially to the renal cortex, and decreased glomerular filtration rate $[11,12]$. In the induction of systemic vasodilatation, which is the primary event in the development of hepatorenal syndrome, systemic endotoxemia is considered to play a pivotal role [12]. In patients with alcoholic hepatitis, the existence and significant role of systemic endotoxemia on liver injury has been shown repeatedly and its pathogenesis has been well described [13, 14]. Alcoholic hepatitis induces a significant increase of intestinal permeability to macromolecules thus permitting the escape of endotoxin from the gut into portal circulation and consequently a depressed elimination of endotoxin by Kupffer cells induces spillover endotoxemia [3, 15]. Processing of endotoxin by Kupffer cells and mainly by extrahepatic macrophages induces cytokinemia [3, 15]. Systemic endotoxemia per se and endotoxin-induced cytokinemia further increase intestinal permeability through modulation of intestinal tight junctions and the vicious cycle of endotoxemia is maintained [16]. In view of this pathophysiological sequence of events, which potential mechanism(s) of action could explain the positive impact of PTX on prevention of hepatorenal syndrome and survival advantage in alcoholic hepatitis?

One potential mechanism could be PTX's positive effect on splachnic and renal microcirculation and this explanation, based on the hemorheological properties of PTX, was proposed in the initial study by Akriviadis et al [8]. However, given the central role of gut-derived endotoxemia in the pathophysiology of hepatorenal syndrome and considering also the significant prevention of hepatorenal syndrome in severe alcoholic hepatitis by PTX administration, as the study by DE BK et al [9] showed total prevention of hepatorenal syndrome, we are tempting to speculate that PTX should inevitably act, at least partly, through reduction of systemic endotoxemia. In other words, PTX might act through maintenance of an effective gut barrier function which prevents endotoxin translocation from gut lumen into portal circulation. Up to now, there is considerable evidence to support this theory. Experimental studies have shown that PTX and its chemically relevant molecule lisofylline effectively prevent intestinal barrier breakdown after various injurious insults like burns, sepsis, hemorrhagic shock or ischemia-reperfusion [17-21]. This protective action on gut barrier function is the result of its regulatory effect on the tight junctions' structure and function, thus leading to preservation of paracellular permeability. Specifically, PTX preserves the expression and cellular distribution of the key tight junction associated molecules occludin and ZO-1, possibly through attenuation of activation of the important tight junction modulator myosin light chain kinase (MLCK) [17-19]. Maintenance of gut barrier function might also be an important part of the positive effect of glucocorticoids in alcoholic hepatitis, beyond their widely accepted mechanisms of action, which is blocking of cytokine release and their potent anti-inflammatory action. Previous studies have demonstrated that glucocorticoids can enhance the intestinal tight junction barrier through inhibition of the TNF- $\alpha$-induced increase in myosin light chain kinase (MLCK) protein expression, a key process mediating the TNF- $\alpha$ increase in intestinal tight junction (TJ) permeability [22]. However, one could ask why the potential prevention of gut barrier function and amelioration of systemic endotoxemia by PTX administration was not accompanied by a concomitant reduction of TNF- $\alpha$ levels and liver injury. One potential answer might be that endotoxin is not the only promoter of TNF- $\alpha$ production in alcoholic hepatitis. Previous studies have shown that there is no correlation between plasma endotoxin and TNF- $\alpha$ levels, whilst lipid peroxidation products and oxygen free radicals are important promoters of TNF- $\alpha$ production as well [23].

In conclusion, we believe that asking "why" in every step of the diagnostic and therapeutic process and attempting to find convincing answers should be a continuous task of all clinicians. Our question on the potential mechanism of action of PTX in severe alcoholic hepatitis, lead us, through a theoretical approach, to reconsider the importance of gut barrier function in the outcome of these patients. Future research may confirm or refute our speculations on PTX's mechanism of action. However, pending convincing answers on this issue, we think that we should augment our focus on protecting our patients' intestinal barrier during hospitalization because of severe alcoholic hepatitis, beyond applying the specific pharmacological strategies of glucocoricoids or PTX. This could be achieved by simple or more specific measures, such as adequate fluid replacement to prevent visceral-microcirculatory disturbances, enteral nutrition to improve microcirculation, prevent mucosal atrophy and provide important nutrients for enterocytes, lactulose administration to reduce intraluminal endotoxin load or by selec- 
tive gut decontamination. In our opinion, understanding in a global way the alterations induced over time in the epithelial cell lining of the intestine in alcoholic hepatitis and discovering selective modulators of intestinal tight junctions or other structures critically involved, in order to control intestinal hyperpermeability, is an emerging and attractive field of research.

\section{Acknowledgements}

The authors declare no commercial associations or conflict of interests related to this article.

\section{References}

1. Mathurin P. Is alcoholic hepatitis an indication for transplantation? Current management and outcomes. Liver Transpl 2005;11:S21-24.

2. Mathurin P, Louvet A, Dharancy S. Treatment of severe forms of alcoholic hepatitis: where are we going? J Gastroenterol Hepatol 2008;23 Suppl 1:S60-62.

3. Fukui H. Relation of endotoxin, endotoxin binding proteins and macrophages to severe alcoholic liver injury and multiple organ failure. Alcohol Clin Exp Res 2005;29:172S-179S.

4. McClain CJ, Barve S, Deaciuc I, Kugelmas M, Hill D. Cytokines in alcoholic liver disease. Semin Liver Dis 1999; 19:205-219.

5. Rongey C, Kaplowitz N. Current concepts and controversies in the treatment of alcoholic hepatitis. World J Gastroenterol 2006;12:6909-6921.

6. McCullough AJ, O'Connor JF. Alcoholic liver disease: proposed recommendations for the American College of Gastroenterology. Am J Gastroenterol 1998;93:20222036.

7. McHutchison JG DR. Pentoxifylline may prevent renal impairment (hepatorenal syndrome) in severe acute alcoholic hepatitis. Hepatology 1991;14:96A.

8. Akriviadis E, Botla R, Briggs W, Han S, Reynolds T, Shakil O. Pentoxifylline improves short-term survival in severe acute alcoholic hepatitis: a double-blind, placebo-controlled trial. Gastroenterology 2000;119:16371648.

9. De BK, Gangopadhyay S, Dutta D, Baksi SD, Pani A, Ghosh P. Pentoxifylline versus prednisolone for severe alcoholic hepatitis: a randomized controlled trial. World J Gastroenterol 2009;15:1613-1619.

10. Shirin H, Bruck R, Aeed H, Frenkel D, Kenet G, Zaidel L, Avni Y, et al. Pentoxifylline prevents concanavalin A-induced hepatitis by reducing tumor necrosis factor alpha levels and inhibiting adhesion of T lymphocytes to extracellular matrix. J Hepatol 1998;29:60-67.

11. Epstein M, Goligorsky MS. Endothelin and nitric oxide in hepatorenal syndrome: a balance reset. J Nephrol
1997; 10:120-135.

12. Dagher L, Moore K. The hepatorenal syndrome. Gut 2001;49:729-737.

13. Parlesak A, Schafer C, Schutz T, Bode JC, Bode C. Increased intestinal permeability to macromolecules and endotoxemia in patients with chronic alcohol abuse in different stages of alcohol-induced liver disease. J Hepatol 2000;32:742-747.

14. Fujimoto M, Uemura M, Nakatani Y, Tsujita S, Hoppo $\mathrm{K}$, Tamagawa T, Kitano H, et al. Plasma endotoxin and serum cytokine levels in patients with alcoholic hepatitis: relation to severity of liver disturbance. Alcohol Clin Exp Res 2000;24:48S-54S.

15. Ishikawa M, Uemura M, Matsuyama $T$, Matsumoto M, Ishizashi $\mathrm{H}$, Kato S, Morioka C, et al. Potential Role of Enhanced Cytokinemia and Plasma Inhibitor on the Decreased Activity of Plasma ADAMTS13 in Patients With Alcoholic Hepatitis: Relationship to Endotoxemia. Alcohol Clin Exp Res 2008.

16. Ma TY, Iwamoto GK, Hoa NT, Akotia V, Pedram A, Boivin MA, Said HM. TNF-alpha-induced increase in intestinal epithelial tight junction permeability requires NF-kappa B activation. Am J Physiol Gastrointest Liver Physiol 2004;286:G367-376.

17. Costantini TW, Loomis WH, Putnam JG, Drusinsky D, Deree J, Choi S, Wolf P, et al. Burn-Induced Gut Barrier Injury Is Attenuated by Phosphodiesterase Inhibition: Effects on Tight Junction Structural Proteins. Shock 2008.

18. Costantini TW, Loomis WH, Putnam JG, Kroll L, Eliceiri BP, Baird A, Bansal V, et al. Pentoxifylline modulates intestinal tight junction signaling after burn injury: effects on myosin light chain kinase. J Trauma 2009;66:17-24; discussion 24-15.

19. Costantini TW, Deree J, Loomis W, Putnam JG, Choi S, Baird A, Eliceiri BP, et al. Phosphodiesterase inhibition attenuates alterations to the tight junction proteins occludin and ZO-1 in immunostimulated Caco-2 intestinal monolayers. Life Sci 2009;84:18-22.

20. Wattanasirichaigoon S, Menconi MJ, Delude RL, Fink MP. Lisofylline ameliorates intestinal mucosal barrier dysfunction caused by ischemia and ischemia/reperfusion. Shock 1999;11:269-275.

21. Wattanasirichaigoon S, Menconi MJ, Fink MP. Lisofylline ameliorates intestinal and hepatic injury induced by hemorrhage and resuscitation in rats. Crit Care Med 2000;28:1540-1549.

22. Boivin MA, Ye D, Kennedy JC, Al-Sadi R, Shepela C, Ma TY. Mechanism of glucocorticoid regulation of the intestinal tight junction barrier. Am J Physiol Gastrointest Liver Physiol 2007;292:G590-598.

23. Bird GL, Sheron N, Goka AK, Alexander GJ, Williams $\mathrm{RS}$. Increased plasma tumor necrosis factor in severe alcoholic hepatitis. Ann Intern Med 1990;112:917-920. 